\title{
Bacillus Cereus Catheter-Related Bacteremia in a Patient Diagnosed with Neuroblastoma
}

\author{
Fatma Köksal Çakırlar1', Nevriye Gönüllü', Şule Çelik¹, Zafer Habip', Gülen Tüysüz², Nuri Kiraz \\ ${ }^{1}$ Department of Medical Microbiology, İstanbul University Cerrahpaşa Faculty of Medicine, İstanbul, Turkey \\ ${ }^{2}$ Department of Pediatrics, İstanbul University Cerrahpaşa Faculty of Medicine, İstanbul, Turkey
}

\begin{abstract}
Bacillus species can be found in air, water, soil, feces, and particularly in the normal flora of patients with a prolonged length of hospitalization. Therefore, isolation of these microorganisms from clinical samples is a common condition and usually regarded as contaminants. Previously, Bacillus cereus has been rarely associated with infections, but in the last few years, it has been reported with increasing frequency as the bacterial pathogen in patients with intravenous drug abuse, trauma, immunodeficiency syndrome, immunosuppressed patients with a history of underlying malignancy and granulocytopenia. In this study, we present a 3-months-old baby girl who was presented to a private health center with a complaint of not sucking well enough. After clinical examination she was diagnosed with neuroblastoma stage $4 \mathrm{~S}$, metastasis was detected in the liver and bone marrow and surrenalectomy and 14 cures of chemotherapy were applied to the patient. Patient was discharged from the hospital with a permanent tunneled catheter, but she returned to the pediatric emergency department with high fever. After laboratory tests she was diagnosed with febrile neutropenia and ceftazidime and amikacin treatment was started. Ceftazidime treatment was stopped after the isolation of $B$. cereus from peripheral and catheter blood cultures of the patient and teicoplanin treatment was started. Permanent tunneled catheter was removed under anesthesia, and this suggested the case as a catheter-originated bacteremia after reducing fever of the patient. As a result, we suggest that B. cereus isolation in blood cultures could not everytime mean contamination, and B. cereus may cause catheter-related infections in immunosuppressed patients. (JAREM 2015; 5: 75-7)
\end{abstract}

Keywords: Bacillus cereus, catheter, bacteremia

\section{INTRODUCTION}

Bacillus cereus is a gram-positive aerobic, spore-forming, rodshaped bacterium that is widely distributed environmentally and is generally regarded as a contaminant (Figure 1). Therefore, isolation of these microorganisms from clinical samples is a common practice. B. cereus is a well-known cause of food poisoning, which is generally benign and self-limiting in a normal host. However, recently, it has been increasingly reported to be a cause of serious and potentially fatal infections in immunosuppressed patients with neutropenia. $B$. cereus has been reported to be a cause of systemic infections such as bacteremia, septicemia, meningitis, respiratory tract infections, surgical wounds, panophthalmitis, pneumonia, and endocarditis among parenteral drug abusers, patients with a suppressed immune system or intraventricular shunt, and newborns (1-5).

In this article, we present a 2-year-old female patient diagnosed with catheter-originated $B$. cereus bacteremia with neuroblastoma stage $4 \mathrm{~S}$

\section{CASE PRESENTATION}

A 3-month-old baby girl patient whose date of birth was 06/09/2010 who had been admitted to a private health center with a complaint of crying and not sucking well enough and had an abdominal ultrasonography showing a left adrenal-originated mass and then admitted to İstanbul University Cerrahpaşa
School of Medicine (I.U.C.S.M.) Pediatric Hematology and Oncology Unit for further examination upon the detection of disseminated metastases in the liver in the abdominal MRI. In the abdominal ultrasonography performed on December 9, 2010, a relatively smooth contoured hyperechoic mass $(46 \times 53 \times 32 \mathrm{~mm}$ in size) on the left adrenal gland and metastases in the liver and bone marrow were detected, and the patient was diagnosed with neuroblastoma stage 4S. In 2011, left surrenalectomy was performed at the Pediatric Surgery Unit of I.U.C.S.M., and 14 cures of chemotherapy were applied to the patient in the Pediatric Hematology and Oncology Unit.

The patient with a permanent tunneled catheter was admitted to the Pediatric Emergency Department of I.U.C.S.M. with high fever on September 6, 2012. Her vital parameters on admission were as follows: blood pressure, $90 / 60 \mathrm{mmHg}$; fever, $38.5^{\circ} \mathrm{C}$; WBC, $2300 / \mathrm{mm}^{3}$; PNL, 300/ $\mathrm{mm}^{3}$; PLT, 21300/ $\mathrm{mm}^{3}$; and hemoglobin, $10.8 \mathrm{~g} / \mathrm{dL}$. The patient was diagnosed with febrile neutropenia. The patient's throat, urine, and blood cultures were collected, and $150 \mathrm{mg} / \mathrm{kg} /$ day of ceftazidime and $15 \mathrm{mg} / \mathrm{kg} /$ day of amikacin were administered. There was no pathogenic bacterium in the throat and urine cultures. Upon the aerobic and gram-positive spore-forming bacillus growth in the blood cultures (BACTEC 9120, Becton Dickinson Diagnostic Systems, Sparks, MD) collected from the three peripheral veins and the catheter, ceftazidime treatment was tapered and $10 \mathrm{mg} / \mathrm{kg} /$ day of teicoplanin was administered (Figure 2). The permanent tunneled catheter 
Table 1. B. cereus susceptibility test: zone diameters and MIC values of various antibiotic

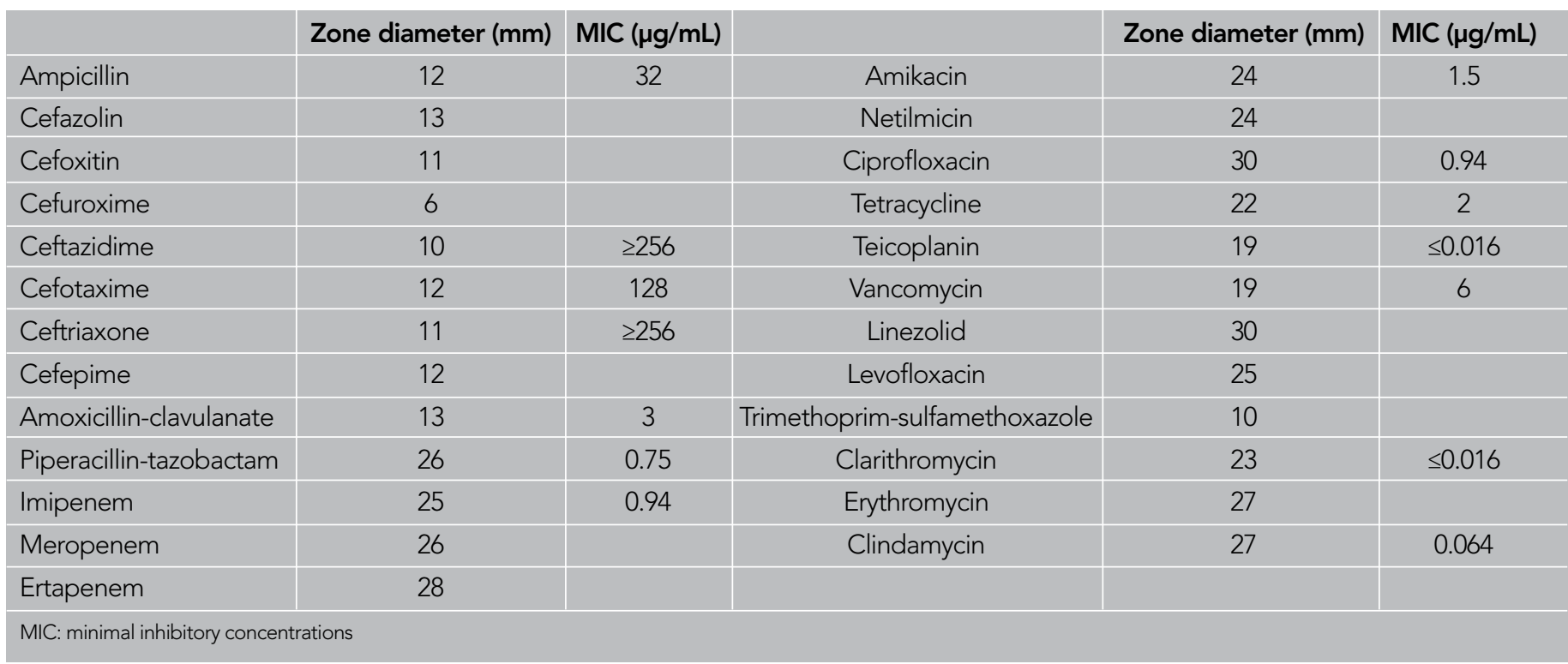

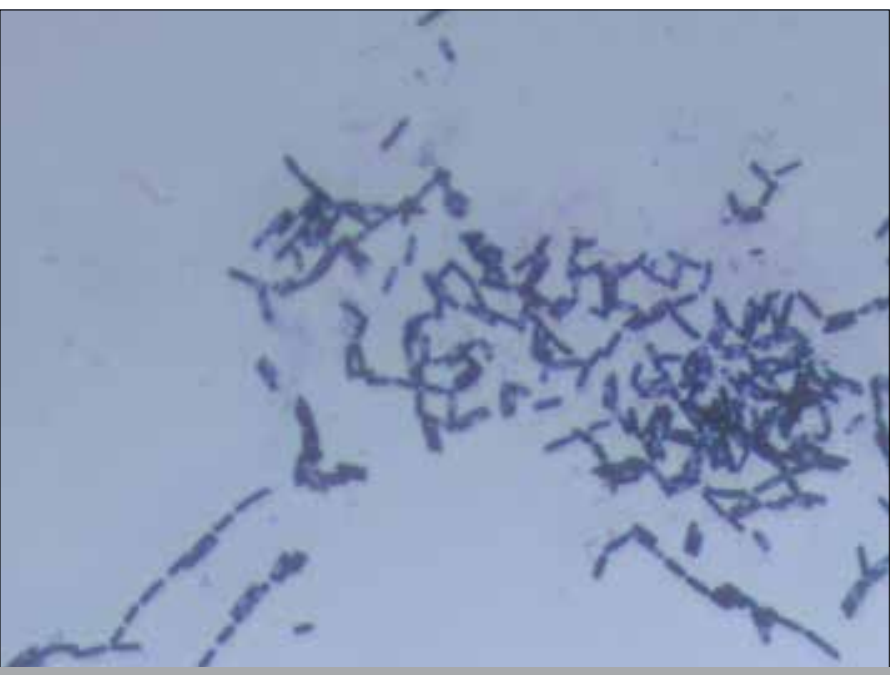

Figure 1. The gram-staining image of $B$. cereus

was removed under general anesthesia, and the patient was discharged because her fever dropped and did not recur, and her general condition was good. This suggested that the bacteremia was a catheter-originated infection.

For bacteria typing in blood cultures, a gram-positive rod panel on an automated identification system Phoenix (Becton Dickinson Diagnostic Systems, Sparks, MD) was used. The result showed a $99 \%$ probability of $B$. cereus. Antibiotic susceptibility test was performed using the Kirby-Bauer method. Because the sensitivity limit values for $B$. cereus were not determined by the Clinical and Laboratory Standards Institute (CLSI) criteria, the zone diameters were expressed only in millimeters (Table 1) (6).

\section{DISCUSSION}

Bacillus species can be found in air, water, soil, feces, and particularly, in the normal flora of patients with a prolonged hospi- talization. Despite its low pathogenicity, it may cause local and systemic infections in addition to food poisoning because of toxins or tissue invasion (1). Previously, B. cereus was rarely associated with infections; however, recently, it has been reported with increasing frequency as the bacterial pathogen in patients with intravenous drug abuse, trauma, immunodeficiency syndrome, immunosuppression and a history of underlying malignancy, and granulocytopenia (7-9). It has been reported that although bacteremia that is caused by $B$. cereus is mostly transient, particularly in patients with a diagnosis of leukemia, it can lead to severe, even fatal, infections such as the fulminant septicemic syndrome $(10,11)$. These infections are mostly catheter-originated. The biofilm layer produced by $B$. cereus is substantially responsible for its adhesion to the catheters; in addition, this layer is the cause of the limited effect of antibiotics by creating a more secure zone for bacteria. Therefore, in $B$. cereus catheter-originated infections, antibiotic therapy alone is generally not sufficient and withdrawal of the catheter is also required $(12,13)$.

B. cereus produces beta-lactamases and is resistant to penicillin, cephalosporin, and trimethoprim-sulfamethoxazole. It is generally sensitive to aminoglycosides, clindamycin, vancomycin, teicoplanin, chloramphenicol, imipenem, and erythromycin. However, despite optimal antibiotic therapy with vancomycin and amikacin, "refractory" B. cereus bacteremia and constantly developing fulminant meningeal infection have been reported in hosts with an immunocompromised immune system (11). In addition, despite being administered treatment comprising multiple antibiotics that included vancomycin, gentamicin, imipenem, and clindamycin, a fatal $B$. cereus infection has been reported in an immunocompromised newborn (14). Although in these cases they seem to be active in vitro against $B$. cereus strains, the selected antimicrobials do not have in vivo activity in some cases. Our female patient who was diagnosed with neuroblastoma stage $4 \mathrm{~S}$ 


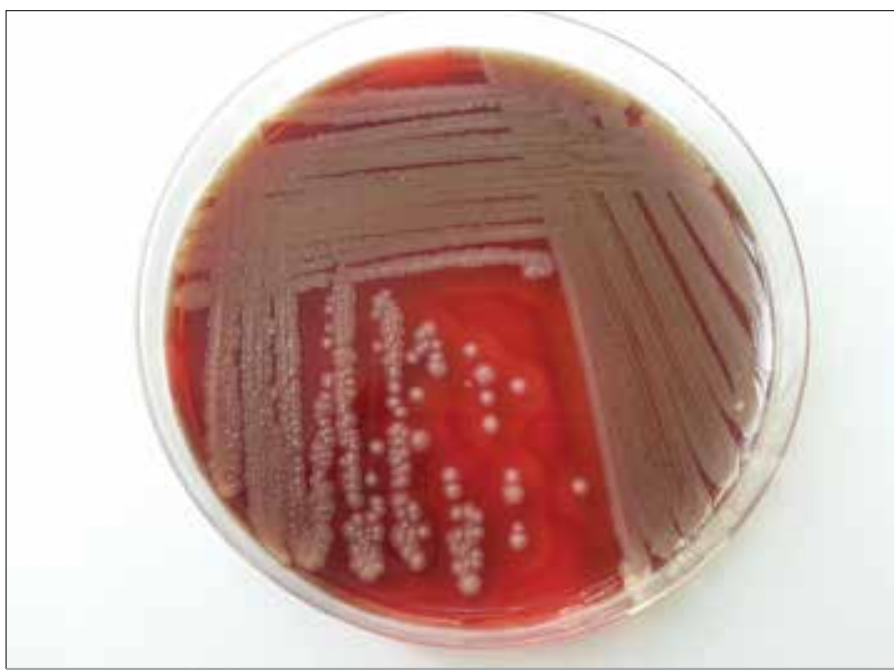

Figure 2. The image of the colonies of $B$. cereus on blood agar plates

after developing bacteremia with $B$. cereus was discharged upon receiving teicoplanin treatment, and her permanent tunneled catheter was removed.

\section{CONCLUSION}

Septicemia caused by $B$. cereus can be fatal in immunosuppressed patients, despite broad-spectrum antibiotic treatment. Thus, it should be considered that $B$. cereus may also be a factor for cases associated with suspected catheter-related infections.

Informed Consent: Written informed consent was obtained from the patient who participated in this case.

Peer-review: Externally peer-reviewed.

Author Contributions: Concept - F.K.Ç., N.G., G.T.; Design - F.K.Ç., N.G., G.T.; Supervision - N.G.; Literature Review - F.K.Ç., Ş.Ç., Z.H.; Writer F.K.Ç.; Critical Review - N.G., G.T., N.K.

Conflict of Interest: No conflict of interest was declared by the authors.
Financial Disclosure: The authors declared that this study has received no financial support.

\section{REFERENCES}

1. Drobniewski FA. Bacillus cereus and related species. Clin Microbiol Rev 1993; 6: 324-38.

2. Thuler LC, Velasco E, de Souza Martins CA, de Faria LM, da Fonseca NP, Dias LM, et al. An outbreak of Bacillus species in a cancer hospital. Infect Control Hosp Epidemiol 1998; 19: 856-8. [CrossRef]

3. Zinner SH. Changing epidemiology of infections in patients with neutropenia and cancer: emphasis on gram-positive and resistant bacteria. Clin Infect Dis 1999; 29: 490-4. [CrossRef]

4. Musa MO, Al Douri M, Khan S, Shafi T, Al Humaidh A, Al Rasheed AM Fulminant septicaemic syndrome of Bacillus cereus: three case reports. J Infect 1999; 39: 154-6. [CrossRef]

5. Sliman R, Rehm S, Shlaes DM. Serious infections caused by Bacillus species. Medicine (Baltimore) 1987; 66: 218-23. [CrossRef]

6. Clinical and Laboratory Standard Institute (CLSI). Performance Standards for Antimicrobial Susceptibility Testing; 20th Informational Supplement. CLSI document M100-S20. CLSI, Wayne, PA: Clinical and Laboratory Standard Institute, 2011.

7. Hernaiz C, Picardo A, Alos JI, Gomez-Garces JL. Nosocomial bacteremia and catheter infection by Bacillus cereus in an immunocompetent patient. Clin Microbiol Infect 2003; 9: 973-5. [CrossRef]

8. Gurler N, Oksuz L, Muftuoglu M, Sargin F, Besisik S. Bacillus cereus catheter related bloodstream infection in a patient with acute lymphoblastic leukemia. Mediterr J Hematol Infect Dis 2012; 4: e2012004. [CrossRef]

9. Banerjee C, Bustamante Cl, Wharton R, Talley E, Wade JC. Bacillus infections in patients with cancer. Arch Intern Med 1988; 148: 1769-74. [CrossRef]

10. Akiyama N, Mitani K, Tanaka Y, Hanazono Y, Motoi N, Zarkovic M, et al. Fulminant septicemic syndrome of Bacillus cereus in a leukemic patient. Intern Med 1997; 36: 221-6. [CrossRef]

11. Ginsburg A, Salazar LG, True LD, Disis ML. Fatal Bacillus cereus sepsis following resolving neutropenic enterocolitis during the treatment of acute leukemia. Am J Hematol 2003; 72: 204-8. [CrossRef]

12. Ash C, Farrow JA, Dorsch M, Stackenbrandt E, Collins MD. Comparative analysis of Bacillus anthracis, Bacillus cereus, and related species on the basis of reverse transriptase sequencing of 16 S rRNA. Int J Syst Bacteriol 1991; 41: 343-6. [CrossRef]

13. Bottone EJ. Bacillus cereus, a volatile human pathogen. Clin Microbiol Rev 2010; 23: 382-98.[CrossRef]

14. Tuladhar R, Patole SK, Koh TH, Norton R, Whitehall JS. Refractory Bacillus cereus infection in a neonate. Int J Clin Pract 2000; 54: 345-7. 